\title{
BENEFÍCIOS DO ISOSTRETCHING SOBRE A FORÇA MUSCULAR RESPIRATÓRIA E A EXPANSIBILIDADE TORÁCICA EM SEDENTÁRIOS
}

Rubian Michelly de Souza, Liamara Basso Dalla Costa e Daniela dos Santos

UNIARP

dani.fisio.santos@gmail.com

Acesso DOI: http://dx.doi.org/10.34059/ciejop.2019v27i1-8

\section{RESUMO}

Objetivos: Examinar o efeito do método sobre a força muscular inspiratória e expiratória, e na expansibilidade torácica de sedentários. Objetivos específicos: explicitar a técnica de Isostretching, classificar a força muscular inspiratória e expiratória, mensurar a expansibilidade torácica em nível axilar, processo xifóide e região basal da população do estudo, e discutir os dados obtidos antes e após a intervenção do método Isostretching. Metodologia: Caracteriza-se como uma pesquisa exploratória, onde 5 voluntários sedentários foram avaliados através do manovacuômetro e da cirtometria e realizaram 20 sessões do método Isostretching. Resultados: Em relação aos valores da PImáx. obteve-se aumento de 41,7\%, e de $25,7 \%$ da PEmáx., enquanto os valores para a expansibilidade torácica revelaram-se de $39 \%$ em nível axilar, $43,9 \%$ no xifoideano e $48,8 \%$ em basal. Conclusão: No estudo apresentado sobre o Isostretching, a técnica aplicada em sedentários durante 20 sessões, obteve dados relevantes sobre a mecânica respiratória.

Palavras-chaves: Isostretching. Força muscular respiratória. Expansibilidade torácica. Sedentarismo.

\section{ABSTRACT}

Objectives: To examine the effect of the method on inspiratory and expiratory muscle strength and on the chest expansion of sedentary patients. Specific objectives: to explain the Isostretching technique, to classify inspiratory and expiratory muscle strength, to measure chest expansion at the axillary level, the xiphoid process and the basal region of the study population, and to discuss the data obtained before and after the Isostretching method intervention. Methodology: It is characterized as an exploratory research, where 5 sedentary volunteers were evaluated through the manovacuometer and the cirtometry and performed 20 sessions of the Isostretching method. Results: In relation to MIP values. $41.7 \%$ and $25.7 \%$ of MEP increased, while values for chest expansion were $39 \%$ at axillary level, $43.9 \%$ at xiphoid and $48.8 \%$ at baseline . Conclusion: In the study presented on Isostretching, the technique applied in sedentary patients during 20 sessions, obtained relevant data on respiratory mechanics. 
Keywords: Isostretching. Respiratory muscle strength. Thoracic expandability. Sedentary lifestyle.

\section{INTRODUÇÃO}

O sedentarismo é caracterizado como a falta ou redução de atividade física (MATTOS, 2006). O estudo das condutas sedentárias tem se tornado relevante, como uma questão de saúde pública (HALLAL et al., 2012). A inatividade física proporciona inúmeros malefícios ao corpo e a saúde, sendo um fator de risco e de predisposição de centenas de doenças tais como obesidade, doenças cardiovasculares, apneia do sono, entre outras (COSTA NETO; PURIFICAÇÃO; LIMA, 2016).

Em contrapartida, a prática de atividade física regular traz benefícios a saúde. Esses efeitos são questionados, seguidamente, entre os profissionais na área da saúde $e$ documentados na literatura atual (FARIAS JUNIOR et al., 2009). A assiduidade de atividade física mantém a força muscular do sistema musculoesquelético e, portanto, tendo influência direta sobre o fortalecimento dos músculos respiratórios. Por conseguinte, sujeitos sedentários apontam déficit na função pulmonar se comparados a indivíduos fisicamente ativos (QUIRINO, 2012).

Diversas técnicas fisioterapêuticas são capazes de promover o treinamento respiratório (SARMENTO, 2009).

O método Isostretching, em particular, não é uma técnica específica para trabalhar a força muscular respiratória, mas apresenta um trabalho respiratório intenso, podendo assim influenciar de maneira positiva na força muscular respiratória. É uma ginástica postural global, criada pelo fisioterapeuta Bernard Redondo, em 1970, na França; um método que educa, corrige e equilibra o corpo, flexibiliza a musculatura, fortalece, melhora a postura e aumenta a capacidade respiratória. A base do método é o auto crescimento da coluna e a expiração forçada (CARVALHO; ASSINI, 2008; JUNIOR et al., 2011).

Trata se de um método postural global, em que os exercícios são executados no maior alinhamento vertebral possível, durante uma longa expiração, com toda a musculatura corporal sendo recrutada (DURANTE; VASCONCELOS, 2009). Seu objetivo pode ser de prevenção ou reabilitação, é praticado por indivíduos de qualquer idade, conforme a capacidade e necessidade de cada um, onde as posturas são realizadas de forma evolutiva, iniciando das mais simples para as mais complexas (REDONDO, 2017). 
Ao presumir que há alterações na força muscular respiratória resultantes do treinamento com o Isostretching, esta pesquisa apresentou como objetivo principal: examinar o efeito do método sobre a força muscular inspiratória e expiratória e na expansibilidade torácica de sedentários; enquanto os objetivos específicos buscaram: explicitar a técnica de Isostretching, classificar a força muscular inspiratória e expiratória dos indivíduos sedentários, mensurar a expansibilidade torácica em nível axilar, processo xifóide e região basal da população do estudo e discutir os dados obtidos antes e após a intervenção da técnica de Isostretching.

\section{METODOLOGIA}

Foram recrutados 12 voluntários, de ambos os sexos, escolhidos aleatoriamente dentre a divulgação realizada na universidade e na comunidade através de folders em Caçador/SC. Os critérios de inclusão foram indivíduo sedentário; idade entre 20 a 45 anos; disponibilidade de horário no período matutino ou noturno; pessoa capaz de compreender comando verbais simples e mais complexos; não ser portador de patologia cardiorrespiratória, neurológica ou musculoesquelética; não possuir deformidades graves, fraturas na coluna e implantes metálicos; sem alterações metabólicas descompensadas; não ser fumante; concordar com a participação mediante a assinatura de um termo de consentimento livre e esclarecido. Como critério de exclusão foram adotados gestantes; faltar em mais de dois atendimentos; indivíduos com alterações cognitivas, que tenham dificuldades na compreensão ou execução das posições. Todos os voluntários receberam informações para a participação no estudo e assinaram um termo de consentimento livre e esclarecido.

O protocolo experimental deste estudo foi aprovado pelo Comitê de ética e Pesquisa envolvendo seres humanos da Universidade Alto Vale do Rio do Peixe- UNIARP através do parecer 2.408.253 em 2018 .

O previsto era atender 10 voluntários em 20 sessões de tratamento, recrutando-se 12 para dar margem a desistências. No entanto, apenas 5 prosseguiram o tratamento (a perda amostral deveu-se a desistência).

A avaliação foi realizada aplicando-se o manovacuômetro, sendo este um instrumento de avaliação da força da musculatura respiratória, mensura pressões positivas (manômetro) e pressões negativas (vacuômetro) (AZEREDO, 2002). É por meio da manovacuometria em que os valores de PImáx e PEmáx são avaliados em centímetros de água (cmH20) (PRESTO; DAMAZIO, 2009). 
A PImáx mede a força dos músculos inspiratórios, e a PEmáx mede dos músculos expiratórios (COSTA, 2004). Os valores são diferentes para homens e mulheres, e o valor ideal varia com a idade (PRESTO; DAMAZIO, 2009). Para Black e Hyatt (1969) apud Presto e Damázio (2009, p. 272), calcula-se os valores de PImáx e PEmáx de acordo com a idade e gênero, assim chegando ao valor previsto de cada indivíduo.

Valores considerados neste estudo segundo tabela de Black e Hyatt(1969):

- Homens (20 a 80 anos): PImáx $(\mathrm{cmH} 2 \mathrm{O})=143-0,55 \times$ Idade

$$
\text { PEmáx }(\mathrm{cmH} 2 \mathrm{O})=268-1,03 \times \text { Idade }
$$

- Mulheres (20 a 80 anos): PImáx $(\mathrm{cmH} 2 \mathrm{O})=104-0,51 \times$ Idade

$$
\text { PEmáx }(\mathrm{cmH} 2 \mathrm{O})=170-0,53 \times \text { Idade }
$$

Todas as medidas foram realizadas com os voluntários sentados, com o tronco em um ângulo de 90 graus com as coxas, os pés apoiados, os membros superiores sem apoio, e a cabeça em posição neutra (CHAO et al., 2010). Para mensuração da PImáx., na posição de sedestação, os voluntários foram instruídos a expirar lentamente, um bocal foi conectado entre seus lábios, com as narinas ocluídas por um clipe nasal, em seguida realizaram uma inspiração máxima, intensa e profunda no manovacuômetro. Para mensuração da PEmáx, os voluntários ainda em posição de sedestação, foram instruídos a uma inspiração lenta até a CPT, após um bocal foi colocado entre os lábios e a via nasal foi ocluída por um clip, uma expiração rápida e intensa foi ser realizada no manovacuômetro (SILVA etal., 2016).

Para cada voluntário foram coletadas três medidas, com um intervalo de 60 segundos de uma aferição para outra, nos casos de erro no processo, a mensuração foi repetida (LANZETTI, 2011). Ao final das três manobras, todas as medidas ficaram registradas na ficha de avaliação, sendo o maior valor escolhido para análise (CARNEIRO et al., 2015). O manovacuômetro utilizado foi Comercial Médica® com escala de 0 a 120 $\mathrm{cmH} 20$.

A cirtometria foi utilizada para avaliar a mobilidade costal e diafragmática, durante os movimentos respiratórios, no repouso, inspiração máxima e expiração máxima. Os perímetros torácicos são mensurados com uma fita métrica (PRESTO; DAMAZIO, 2009). $\mathrm{Na}$ avaliação da mobilidade torácica as medidas acontecem em três regiões. No perímetro axilar (PA) a fita passa pelos cavos axilares ao nível da $3^{\underline{a}}$ costela, no perímetro xifoide (PX) a fita passa sobre o apêndice xifoide ao nível da $7^{\text {a }}$ cartilagem costal e no perímetro basal (PB) a fita passa sobre as $12^{\mathrm{a}}$ costelas (LEHMKUHL et al., 2005). 
Os valores de referência da cirtometria segundo Lianza (1995) apud Santos (2012, p. 26). Muito reduzido: menor que 2 centímetros, reduzido: de 2 a 4 centímetros e normal: maior que 4 centímetros.

\section{Protocolo de Tratamento}

A técnica Isostretching foi aplicada em 20 sessões de 50 minutos, 2 vezes por semana como recomenda o autor da técnica. As posturas aplicadas no protocolo de tratamento foram baseadas nas técnicas descrita por Redondo (2017) em seu livro ISOSTRETCHING Método de correção postural.

A execução dos exercícios de foram realizadas da seguinte ordem: Posição: é a postura de partida com tomada de referência; Correção: alinhamento da coluna e da pelve. É a posição que controla as compensações; Ação: é a junção dos diversos elementos: Contração isométrica, abaixamento das escápulas (fixação isométrica), auto crescimento da coluna vertebral e a expiração profunda e prolongada. No instante da posição correta, faz-se a inspiração. Durante o auto crescimento, expira-se de forma controlada e prolongada, mantendo a postura. O tempo mantido da postura é administrado pela expiração profunda e prolongada. Tempo esse que é a proporção em que a pessoa consegue manter a postura. Foram alternadas as sequências de alongamentos e de recuperação.

Inicialmente foi realizado aquecimento ativo, movimentos de baixa intensidade, apenas para elevação da temperatura corporal e aquecimento dos tecidos, com objetivo de preparar os voluntários para os exercícios, melhorando a mobilidade das articulações, melhorando da flexibilidade, dessa forma facilitando as posturas (KNUDSON, 2008; WEINECK, 2003). O tempo foi distribuído da seguinte maneira:5 minutos de aquecimento;10 minutos na posição em pé;20 minutos na posição sentado; 10 minutos na posição deitado. Cada postura foi mantida durante três expirações forçadas, a partir da posição correta, sendo repetidas duas vezes com intervalo de um minuto entre a mudança de postura.

\section{RESULTADOS E DISCUSSÃO}

Apenas 5 voluntários completaram o tratamento com 20 sessões, sendo estes 3 mulheres e 2 homens, com média de idade 34,8 $\pm 8,9$ anos. 
Gráfico 01 - Comparativo da PImáx. antes e após intervenção do método Isostretching através da manovacuometria

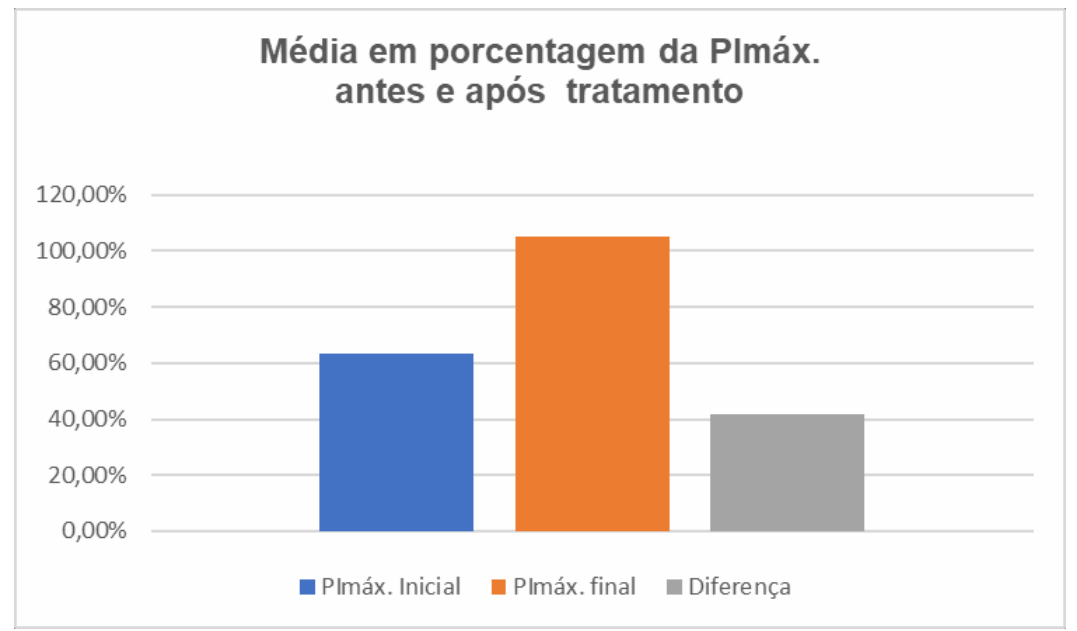

Fonte: Dados da pesquisa, 2018.

A média inicial de PImáx da amostra foi de $\left(-63 \pm 8,4 \mathrm{cmH}_{2} \mathrm{O}\right)$ equivalente a $63,4 \%$ do valor ideal. Após o tratamento, a média da PImáx aumentou para $\left(-105 \pm 10 \mathrm{cmH}_{2} \mathrm{O}\right)$ equivalente a $105,1 \%$ do previsto, demonstrando uma diferença de $41,7 \%$ na força da musculatura inspiratória dos participantes.

Os métodos de correção postural, de acordo com a literatura, favorecem a respiração, o alinhamento da postura sucede maior desenvoltura da mecânica respiratória. O Isostretching usado, além de promover a correção postural tem o uso constante da musculatura respiratória, oferecendo benefícios aos indivíduos praticantes da técnica (ALEXANDER, 1977; YOKOHAMA, 2004). 
Gráfico 02 - Comparativo da PEmáx antes e após intervenção do método Isostretching através da manovacuometria

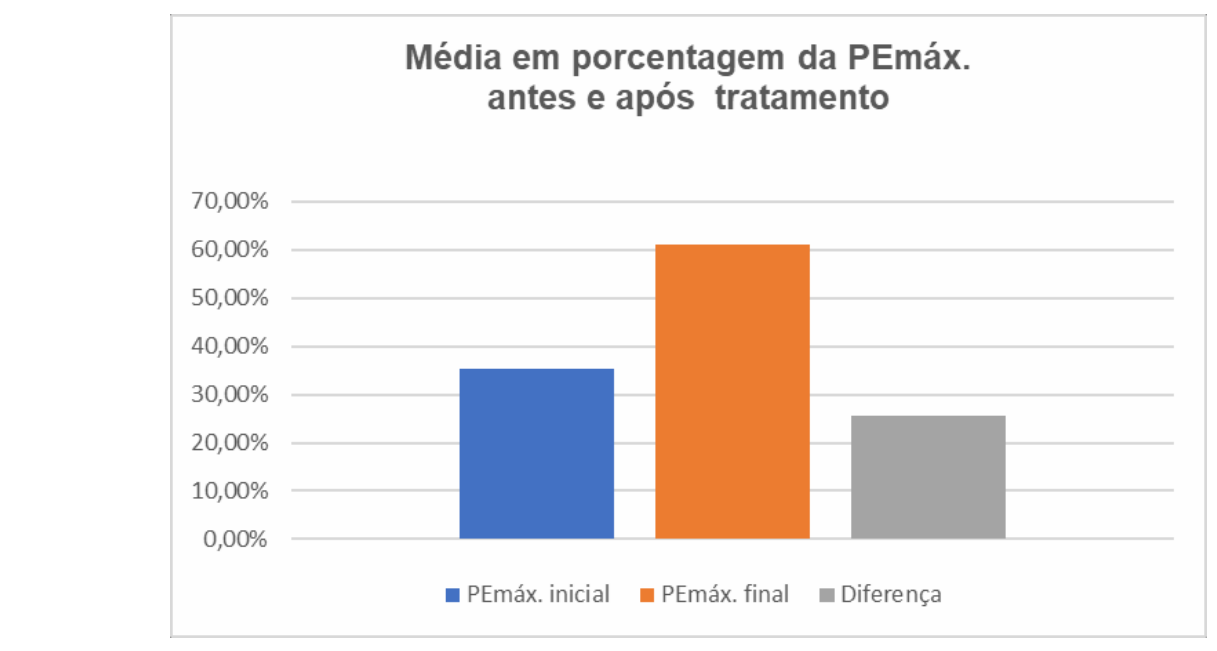

Fonte: Dados da pesquisa, 2018.

A média inicial da PEmáx. da amostra foi de $\left(+61 \pm 14,3 \mathrm{cmH}_{2} \mathrm{O}\right)$, equivalente a $35,4 \%$ do valor ideal. Após o tratamento, a média da PEmáx. aumentou para $\left(+108 \pm 4,5 \mathrm{cmH}_{2} \mathrm{O}\right)$, equivalente a $61,1 \%$ do previsto, demonstrando uma diferença de $25,7 \%$ na força da musculatura expiratória dos sedentários.

$\mathrm{Na}$ primeira avaliação da força muscular respiratória, mensurada através da manovacuometria, verificou-se que todos os participantes sedentários apresentavam força respiratória abaixo do valor previsto para sua idade. A força respiratória baixa ou no limite inferior da normalidade, pode ser consequência de diversos fatores como idade, sexo, altura, obesidade e tabagismo (BESSA; LOPES; RUFINO, 2015).

Ao examinar os gráficos 1 e 2 nos resultados da PImáx. e PEmáx. identificou- se uma melhora na função dos músculos respiratórios através da técnica Isostretching. Como aconteceu no estudo de Freire, Ribeiro e Zago (2016) onde os participantes apresentaram aumento da força respiratória e os autores sugeriram que o Isostretching pode alterar a força muscular respiratória em ambos os sexos.

Rezende et al., (2016) realizaram um estudo com 17 pré-adolescentes, em 12 sessões de Isostretching com duração de uma hora, que demonstrou ser suficiente para 
melhorar a capacidade respiratória, os valores foram obtidos através do peak flow e manovacuômetro.

Quinderé, Silva e Mont'Alverne (2012) em um estudo com 3 idosas, em 10 sessões de Isostretching, duas vezes por semana com duração de meia hora, tiveram resultados de evolução importante na função pulmonar obtidos através da espirometria.

Em um estudo associando a técnica de Isostretching e pompage realizado por Pasqualoto et al., (2013), cinco indivíduos com doença pulmonar obstrutiva crônica, realizaram o tratamento em quatro semanas de intervenção das técnicas, e obtiveram resultados de aumento de força muscular inspiratória e expiratória nos cinco participantes, aumento da distância percorrida no TC6 e aumento na expansibilidade torácica.

Os resultados obtidos na cirtometria de todos os voluntários da pesquisa, antes e após a aplicada da técnica de Isostretching, estão apresentados no gráfico 3.

Gráfico 3 - Comparativo da cirtometria torácica, realizada nas regiões axilar, xifoide e basal antes e após o método Isostretching.

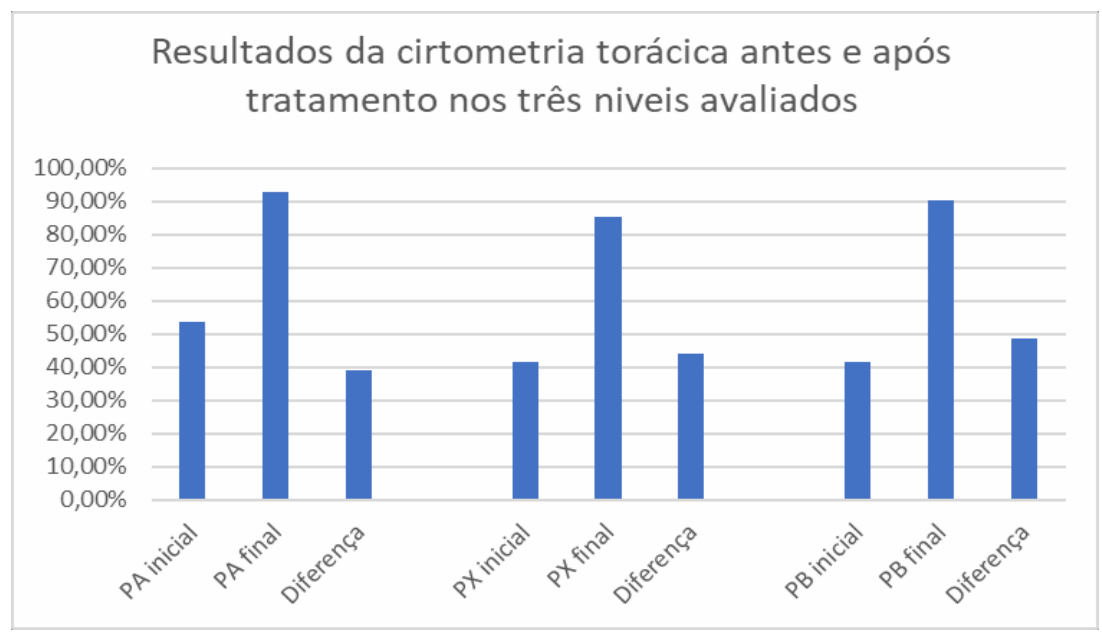

Fonte: Dados da pesquisa, 2018.

A mobilidade torácica em nível axilar teve uma média de $(2,2 \pm 0,27 \mathrm{~cm})$, considerada uma mobilidade reduzida, sendo apenas $53,7 \%$ do valor da normalidade. A avaliação realizada após o tratamento atingiu um valor de $(3,8 \pm 0,45 \mathrm{~cm})$, equivalente a $92,7 \%$, este aumento de $39 \%$ aproximou-se do padrão de normalidade.

Em relação à mobilidade torácica em nível de processo xifoide a média foi de $(1,7 \pm 0,45 \mathrm{~cm})$, considerada uma mobilidade muito reduzida, pois equivale a $41,5 \%$ da normalidade. Na reavaliação o valor foi de $(3,5 \pm 0,61 \mathrm{~cm})$, correspondente a $85,4 \%$, mesmo com $43,9 \%$ de acréscimo o valor não alcançou os valores normais. 
Para a mobilidade torácica em nível basal a média inicial foi de $(1,7 \pm 0,84 \mathrm{~cm})$, considerada uma mobilidade muito reduzida, 'sendo apenas $41,5 \%$ do valor esperado. $\mathrm{Na}$ avaliação pós tratamento o valor passou para $(3,7 \pm 0,57 \mathrm{~cm})$, equivalente a $90,3 \%$ do valor previsto. Com essa evolução de $48,8 \%$ confere a este o maior ganho, aproximando-se do padrão da normalidade.

A mobilidade torácica a nível axilar inicialmente era reduzida, e na reavaliação apesar do ganho, se manteve reduzida, mas próximo do valor da normalidade. Enquanto em processo xifoide e basal, inicialmente foram classificados como uma mobilidade muito reduzida, e passaram para nomeação de reduzida. Vemos um aumento em todos os níveis avaliados, axilar, xifoide e basal, com maior significância na região basal.

A mobilidade torácica está ligada ao processo respiratório, sendo que a cada respiração ocorre movimento na caixa torácica, na inspiração expandindo-se e na expiração retraindo-se (LOPEZ, MEDEIROS, 2001). A região inferior da caixa torácica é mais móvel que a superior, portanto a expansão do tórax é influenciada pelo comprimento das cartilagens costais da qual vai aumentando da $1^{\circ}$ a $10^{\circ}$ costela (TRIBASTONE, 2001).

Ao comparar os valores das mensurações inicias da cirtometria torácica, com os resultados obtidos ao final de 20 sessões de Isostretching, os efeitos do tratamento proposto mostram-se positivos como no estudo feito por Batista e Lima (2008) que constata que ao praticar o Isostretching, o método causa alterações na mobilidade toracoabdominal.

Yokohama (2004) em seu estudo com 3 mulheres, em 10 sessões de Isostretching, 2 vezes por semana, com duração de 50 minutos, obteve resultados relevantes sobre a mecânica respiratória, indicando o método proposto como um tratamento eficiente no aumento da expansibilidade toracopulmonar.

No estudo realizado por Burkle, Shiraishi e Yabiku (2008) em pacientes mastectomizadas com series de casos realizado durante um mês, três vezes por semana com duração de 45 minutos, o método do Isostretching ao final de 12 sessões ganhou benefícios em todos os itens avaliados: tanto na função pulmonar avaliada através da espirometria, expansibilidade torácica através da cirtometria e aumento da ADM do ombro através da goniometria.

Similar ao estudo acima a intervenção com o método Isostretching nesse trabalho também promoveu aumento da PImáx em $41,7 \%$ como mostra o gráfico 1 , e de $25,7 \%$ da PEmáx, conforme mostra o gráfico 2. Promoveu ainda, aumento relação à expansibilidade torácica, nas medidas dos três níveis avaliados na cirtometria, sendo $39 \%$ no axilar, 43,9\% no xifoideano e $48,8 \%$ no basal, como mostra o gráfico 3 . 
Considerando as literaturas comparadas há efetividade do método utilizado. De acordo com os resultados, observa-se que a técnica de Isostretching aplicada em sedentários foi eficaz no aumento da força muscular inspiratória e expiratória, como também no aumento da mobilidade torácica.

\section{CONCLUSÃO}

O estudo apresentado sobre a técnica do Isostretching, mostra que ela aplicada em sedentários durante 20 sessões, obteve dados relevantes sobre a mecânica respiratória, mostrando-se um método não apenas de correção postural, mas eficaz na melhora da força respiratória, como também no aumento da expansibilidade torácica dos indivíduos submetidos a ela.

Além desses resultados, outros benefícios além dos objetivos do estudo foram relatados pelos participantes da pesquisa. Devido ao método trabalhar de uma forma global, ele interferiu na melhora da flexibilidade, na resistência, no aperfeiçoamento deles no decorrer dos atendimentos e ainda na qualidade do sono.

\section{REFERÊNCIAS}

ALEXANDER, L. O corpo em terapia. São Paulo: Summers, 1977.

ANDRADE, E. A; PINTO, A. J. S; ANDRADE, A. Estados de humor e estilo de vida de idosos ativos e sedentários. Revista Brasileira de Prescrição e Fisiologia do Exercício (RBPFEX), v. 2, n. 7, p. 10, 2008.

AZEREDO, C. A. C. Fisioterapia respiratória moderna. 4. ed. São Paulo: Manole, 2002.

BATISTA, J. B. S; LIMA, M. C. O iso-stretching no ganho de expansibilidade torácica e força muscular respiratória em indivíduos saudáveis. II Seminário de Fisioterapia da UNIAMERICA: Iniciação Científica, 5 e 6 de maio 2008. Foz do Iguaçu.

BESSA, E. J. C; LOPES, A. J; RUFINO, R. A importância da medida da força muscular respiratória na prática da pneumologia. Pulmão RJ, v. 24, n. 1, p. 3741, 2015.

BLACK, L. F; HYATT, R. E. Maximal respiratory pressures: normal values and relationship to age and sex. American Review of Respiratory Disease, v. 99, n. 5, p. 696-702, 1969. 
BURKLE, A. B; SHIRAISHI, F. O; YABIKU, F. N. Aplicação do método Isostretching em pacientes mastectomizadas: série de casos. Saúde e Pesquisa, v. 1, n. 2, p. 161-166, 2008.

CARNEIRO, R. C. M. et al. Estudo da força muscular respiratória em pacientes submetidos à cirurgia cardíaca em um hospital na cidade de Fortaleza/CE. Journal of Health Sciences, v. 15, n. 4, 2015.

CARVALHO A. R; ASSINI, T. C. K. A. Aprimoramento da capacidade funcional de idosos submetidos a uma intervenção por isostretching. Revista Brasileira de Fisioterapia, v. 12, n. 4, 2008.

CHAO, A. C. et al. Efeito da reeducação postural global na força muscular respiratória em indivíduos saudáveis. Curso de Fisioterapia da Universidade Estadual do Oeste do Paraná. V congresso paranaense de fisioterapia, 2010.

COSTA NETO, F. N; PURIFICAÇÃO, T. A; LIMA, U. D. A. Influência das novas tecnologias no sedentarismo atual. In: Encontro Regional dos Estudantes de Educação Física, v. 1, n. 1, 2016, João Pessoa. Anais Eletrônicos. João Pessoa: UFPB, 2016, p. 01-11. Disponível em: $<$ https://anais.meefpe.org/index.php/ereef/article/view/84/23> Acesso em: 05 jun. 2017.

DURANTE, H; VASCONCELOS, E.C.L.M. Comparação do método Isostretching e cinesioterapia convencional no tratamento da lombalgia. Semina: Ciências Biológicas e da Saúde, v. 30, n. 1, p. 83-90, 2009.

FARIAS JÚNIOR, J. C. et al. Comportamentos de risco à saúde em adolescentes no Sul do Brasil: prevalência e fatores associados. Revista Panamericana de Salud Pública, v. 25, p. 344-352, 2009.

FREIRE, E; RIBEIRO, A. S. C; ZAGO, L. B. Fortalecimento muscular e expansibilidade pulmonar após o uso da técnica de Isostretching. Interfaces Científicas-Saúde e Ambiente, v. 4, n. 3, p. 31-38, 2016.

HALLAL, P. C. et al. Physical activity: more of the same is not enough. The Lancet, v. 380, n. 9838, p. 190-191, 2012.

Isostretching no incremento da função pulmonar de Gerontos. Anais da Jornada de Fisioterapia da UFC, v. 1, n. 1, p. 44, 2012.

KNUDSON, D. V. Warm-up and Flexibility. In: Chandler TJ, Brown LE. Conditioning for strength and human performance. Philadelphia, PA: Lippincott-Williams \& Wilkins; 2008. 
LANZETTI, C. E. Analise da força muscular respiratoria em pneumopatas cronicos participantes do programa de reabilitação pulmonar. Revista Científica do Unisalesiano - Lins - SP, ano 2, n.4, jul/dez de 2011.

LEHMKUHL, E. et al. A mobilidade torácica avaliada em diferentes regiões através da técnica de cirtometria em indivíduos saudáveis. 2005. Graduação de Fisioterapia. Universidade do Vale do Itajaí - UNIVALI.

LIANZA, S. Medicina de reabilitação. 2. ed. Rio de Janeiro: Guanabara Koogan, 1995.

LOPEZ, M; MEDEIROS, I.L. Semiologia médica: as bases do diagnóstico clínico. 4 ed. Rio de Janeiro: Revinter; 2001.

MATTOS, A. D. et al. Atividade física na sociedade tecnológica. Revista Digital EFDeportes. com, p. 24-29, 2006.

Monografias Do Curso De Fisioterapia - UNIOESTE n. 01-2004 ISSN 1678-8265. Cascavel, 2004. Disponível em: <http://www.unioeste.br/projetos/ezlrf/ monografias/2004-1/tcc/pdf/thama.PDF>. Acesso: 15 set. 2018.

OLIVEIRA, K. M. et al. Respiratory muscle strength and thoracic mobility in children and adolescents with acute leukemia and healthy school students. Rev Bras Cancerol, v. 57, p. 511-7, 2011.

PASQUALOTO, A. S. et al. Análise dos desfechos das técnicas de Isostretching e Pompage em pacientes com doença pulmonar obstrutiva crônica. Saúde (Santa Maria), v. 39, n. 2, p. 161-170, 2013.

PRESTO, B; DAMAZIO, L. Fisioterapia na UTI. Rio de Janeiro: Elsevier, 2. ed, 2009.

QUINDERÉ, M. L; SILVA, S. A; MONT’ALVERNE, D. G. B. A influência do método QUIRINO, C. P. et al. Efeitos de um protocolo de exercícios baseados no método Pilates sobre variáveis respiratórias em uma população de jovens sedentários. Physical Therapy Brazil, v. 13, n. 2, p. 124-132, 2012.

REDONDO, B. Isostretching: método de correção postural- São Paulo- Andreoli, 2017.

REZENDE, M. et al. Efeitos do método Isostretching na função respiratória e postura de respiradores bucal. ConScientiae Saúde, v. 15, n. 1, 2016.

SARMENTO, G. J. V. O ABC da fisioterapia respiratória. 01. ed. São Paulo: Manole, 2009. 
SILVA, C. M. et al. Avaliação da força muscular respiratória e capacidade funcional em pacientes com fibrose cística. Acta Fisiátrica, v. 23, n. 4, p. 186190, 2016.

TRIBASTONE, F. Tratado de exercícios corretivos- Aplicados à reeducação motora e postural. SP: Manole, 2001.

WEINECK J. Treinamento Ideal. 9.ed. São Paulo: Manole, 2003.

YOKOHAMA, T. V. A prática do Isostretching na melhora da expansibilidade toracopulmonar, verificada através da espirometria e da cirtometria. Disponível em: <https://studylibpt.com/doc/676649/efeito-da- reeduca\%C3\%A7\%C3\%A3opostural-global-na-for\%C3\%A7a-muscular>. Acesso em: 12 jun. 2017. 